Subscriber access provided by University of East Anglia Library

\title{
Article
}

\section{The Crystal Structure of the Transcription Regulator RsrR Reveals a [2Fe-2S] Cluster Coordinated by Cys, Glu and His Residues.}

Anne Volbeda, Ma Teresa Pellicer Martinez, Jason C. Crack, Patricia Amara, Océane Gigarel, John T. Munnoch, Matthew I. Hutchings, Claudine Darnault, Nick E. Le Brun, and Juan C. Fontecilla-Camps

J. Am. Chem. Soc., Just Accepted Manuscript • DOI: 10.1021/jacs.8b10823 • Publication Date (Web): 18 Jan 2019

Downloaded from http://pubs.acs.org on January 22, 2019

\section{Just Accepted}

"Just Accepted" manuscripts have been peer-reviewed and accepted for publication. They are posted online prior to technical editing, formatting for publication and author proofing. The American Chemical Society provides "Just Accepted" as a service to the research community to expedite the dissemination of scientific material as soon as possible after acceptance. "Just Accepted" manuscripts appear in full in PDF format accompanied by an HTML abstract. "Just Accepted" manuscripts have been fully peer reviewed, but should not be considered the official version of record. They are citable by the Digital Object Identifier (DOI®). "Just Accepted" is an optional service offered to authors. Therefore, the "Just Accepted" Web site may not include all articles that will be published in the journal. After a manuscript is technically edited and formatted, it will be removed from the "Just Accepted" Web site and published as an ASAP article. Note that technical editing may introduce minor changes to the manuscript text and/or graphics which could affect content, and all legal disclaimers and ethical guidelines that apply to the journal pertain. ACS cannot be held responsible for errors or consequences arising from the use of information contained in these "Just Accepted" manuscripts. 


\section{INTRODUCTION}

Proteins from the Rrf2 superfamily of dimeric bacterial transcription factors known or predicted to coordinate [Fe-S] clusters are diverse. They include NsrR that senses nitric oxide (NO), RirA which detects iron limitation ${ }^{1}$ and IscR which senses the Fe-S cluster status of the cell. ${ }^{2}$ The X-ray structures of Escherichia coli $(E c)$ apo-IscR, alone and in complex with DNA, ${ }^{3,4}$ and of Streptomyces coelicolor $(S c)$ holo-NsrR have already been reported, the latter by us. ${ }^{5}$ Despite the structural similarity between different [Fe-S] cluster-binding Rrf2 proteins, the type of cluster is variable, with both $[4 \mathrm{Fe}-4 \mathrm{~S}]$ (e.g. NsrR) and $[2 \mathrm{Fe}-2 \mathrm{~S}]$ (e.g. IscR) clusters known. The cluster coordination also appears to be variable but is believed to involve three conserved Cys residues. ${ }^{6-8}$

A very recently discovered member of the Rrf2 family is the dimeric $\underline{R} e d o x$ sensitive response $\underline{\text { Regulator RsrR that appears }}$ to sense the redox status of the cell via a [2Fe-2S] cofactor contained in each of its monomers. ${ }^{9}$ In the recombinant asisolated Streptomyces venezuelae $(S v)$ RsrR the [2Fe-2S] cluster switches readily between oxidized $(+2)$ and reduced $(+1)$ states. This switch controls its DNA binding activity, with the oxidized holo-SvRsrR showing highest affinity for its nucleic acid sites. Although exposure to $\mathrm{O}_{2}$ is sufficient to cause oxidation, it is possible that, in vivo, other oxidants can also react with RsrR.

Using ChIP-seq and ChIP-exo analyses some of us identified 630 RsrR binding sites on the S. venezuelae genome and we grouped them into two classes. ${ }^{9}$ The class 1 binding sites, of which there are 14, consist of an 11-3-11 base pairs inverted repeat motif. The remainder are all class 2 binding sites, which have only a single motif (half site). Examination of class 1 target genes suggests that $S v$ RsrR plays a primary role in regulating the relative concentrations of NADH and $\mathrm{NAD}(\mathrm{P}) \mathrm{H}$. Indeed, in the $\Delta r s r R$ mutant the most highly induced transcript is that from sven6562, a gene that encodes a putative $\mathrm{NAD}(\mathrm{P})^{+}$ binding repressor of the nitrogen metabolite repression (NmrA) family. ${ }^{9}$ Consequently, we have proposed that reduction of holo- $S v$ RsrR induces expression of this gene, the product of which senses the redox status of the cell via the NAD $(\mathrm{P})^{+}$-to$\mathrm{NAD}(\mathrm{P}) \mathrm{H}$ concentration ratio. The sven 6562 gene product then modulates expression of its own regulon, which probably overlaps with that of RsrR. In addition, six RsrR targets are known to be involved in glutamate and glutamine metabolism. ${ }^{9}$ Importantly, these amino acids are precursors in the synthesis of 1D-myo-inosityl 2-(N-acetyl-L-cysteinyl)amido-2-deoxyalpha-D glucopyranoside (called mycothiol or MSH) which is the equivalent of glutathione in Actinobacteria.${ }^{10}$ Besides acting as a reducing agent, mycothiol functions in cysteine storage and is involved in the detoxification of redox species and antibiotics. ${ }^{10}$ Thus $S v$ RsrR seems to be involved in the control of the redox state of the cell in more than one way.

The properties of $S v$ RsrR are reminiscent of those of the E. coli [2Fe-2S] cluster-containing transcription factor SoxR. ${ }^{11}$ However, reduction of $S v$ RsrR greatly diminishes its affinity for DNA rather than modulating the distortion of the nucleic acid 
like reduced SoxR has been proposed to do. ${ }^{12}$ Furthermore, a truncated SoxR lacking its [2Fe-2S] cluster can still bind DNA ${ }^{13}$ which is not the case for the cluster-free form of SvRsrR. ${ }^{9}$ This latter observation underscores the central role the cluster plays in generating the active form of $S v R s r R$ and raises the question of how a simple one electron redox process can generate the conformational changes necessary to activate or abolish DNA-binding.

Previous studies revealed that the absorbance properties of [2Fe-2S] RsrR are highly characteristic of a cluster with His coordination ${ }^{9}$ and are markedly oxidation state-dependent: the oxidized, [2Fe-2S $]^{2+}$ cluster is brown $\left(\lambda_{\max } 460 \mathrm{~nm}\right)$ and the reduced, $[2 \mathrm{Fe}-2 \mathrm{~S}]^{1+}$ cluster is pink $\left(\lambda_{\max } 540 \mathrm{~nm}\right)$. The latter is also paramagnetic $(S=1 / 2)$, giving rise to an EPR spectrum characteristic of a $[2 \mathrm{Fe}-2 \mathrm{~S}]^{1+}$ cluster. The cluster could be readily cycled between oxidation states, with oxidation achieved rapidly upon exposure to $\mathrm{O}_{2}$ and reduction via addition of a 1:1 ratio of dithionite (DTH). ${ }^{9}$

\section{DSSP LLLLLHHHHH-HHHHHHHHLL---LLLLLHHHHH- RSrR MKLSGGVEWA-LHCCVVLTAA---SRPVPAARIA- ISCR MRLTSKGRYA-VTAMLDVALNSE-AGPVPLADIS- NSrR MRLTKFTDLA-LRSLMRLAVVRDGDEPLATREVA-}

DSSP HHLLLLHHHH-HHHHHHHHHL-LLEEEELLLL-

RsrR ELHDVSPSYL-AKQMQALSRA-GLVRSVQGKT-

ISCR ERQGISLSYL-EQLFSRLRKN-GLVSSVRGPG-

NSrR EVVGVPYTHA-AKAITRLQHL-GVVEArrgrg-

DSSP LEEEELLLHH-HLLHHHHHHH-HHLLLLLLLL-

RsrR GGYVLTRPAV-EITLLDVVQA-VDGPDPAFVC-

IscR GGYLLGKDAS-SIAVGEVISA-VDesvdatrc-

NsrR gGLTLTDLGR-RVSVGWLVRE-LEGEAEVVDC-

DSSP ----LLHHhlllll-llhhhlLLLL-HHHHHHHHHH-

RsrR ---TEIRQRGPLA-TPPEKCTKAC-PIARAMGAAE-

IscR qgkggcqg--------------gdkC-LTHALWRDLS-

NsrR EGDNPCPL-----------RGAC-RLRRALRDAQ-

DSSP HHHHHHHHHL-LHHHHHHHHH-HHIlllhhhhhhhhhhll RSrR AAWRASLAAT-TIADLVATVD-DESGPDALPGVGAWLIEG IsCR DRLTGFLNNI-TLGELVNNQE-VL--------------NsrR EAFYAALDPL-TVTDLVAAPT-----gPVLLGLTD-----

Figure 1. Amino acid residue sequence comparison of $S v$ RsrR with the Rrf2 family members $E c$ IscR and $S c$ NsrR based on their structural superposition with the DALI server. ${ }^{14}[\mathrm{Fe}-\mathrm{S}]$ cluster ligands are highlighted in green, conserved residues are shown in bold and structurally non-resolved residues are depicted in lower case. Secondary structure defined by $\mathrm{DSSP}^{15}$ is indicated as helix $(\mathrm{H})$, sheet $(\mathrm{E})$ and coil $(\mathrm{L})$, using lower case letters for nonconserved regions.

Here we report three structures of wild-type holo- $S v$ RsrR: one from an air-treated dark brown crystal (RsrR-2), one from a pale brown crystal (RsrR-3), which is assumed to be partially reduced) and another from a DTH-treated pink crystal (RsrR-4) and at $1.6 \AA, 2.0 \AA$, and $2.3 \AA$ resolution, respectively (Table S1 and Figure S1). Unexpectedly, none of Cys13, Cys14 or Cys 106 is a $[2 \mathrm{Fe}-2 \mathrm{~S}]$ cluster ligand. Instead, this cluster is coordinated by Glu8 and His 12 from one monomer and Cys 90 and Cys110 from the other (Figure 1). This is the first known case of coordination of a biological $[\mathrm{Fe}-\mathrm{S}]$ cluster by three different types of protein ligands and, to the best of our knowledge, this is the first time a glutamate residue is found to coordinate a $[2 \mathrm{Fe}-2 \mathrm{~S}]$ cluster. Interestingly, and depending on the space group, the neighboring Trp9 side chain can adopt either a solvent-exposed conformation, here called $\mathrm{W}_{\mathrm{o}}$, or a buried one called $\mathrm{W}_{\mathrm{i}}$ where it occupies a pre-existing protein cavity.

\section{RESULTS}

Structure of the holo-SvRsrR homodimer reveals unprecedented [2Fe-2S] cluster coordination

The three-dimensional structure of $S v R$ RrR was solved by single anomalous dispersion (SAD) phasing at $2.0 \AA$ resolution using an anaerobically grown monoclinic $\left(\mathrm{P} 2_{1}\right)$ crystal from a 96-well plate set up with a Gryphon robot (Art Robins Inst. CA, USA) with the vapor diffusion method. X-ray data were collected at the Fe edge ( $\lambda=1.73978 \AA$, column RsrR-1 in Table S1; see Methods). Another crystal from the same crystallization drop was taken out of the glove box, stabilized in an aerated cryoprotectant solution and flash-cooled after $10 \mathrm{~min}$ of exposure to air and stored in liquid $\mathrm{N}_{2}$. X-ray data were collected from this crystal, which had a dark brown color indicative of cluster oxidation (Figure S1A), to $1.6 \AA$ resolution at $\lambda=0.94500 \AA$ (RsrR-2 in Table S1). These data were used for crystallographic refinement (see Methods).

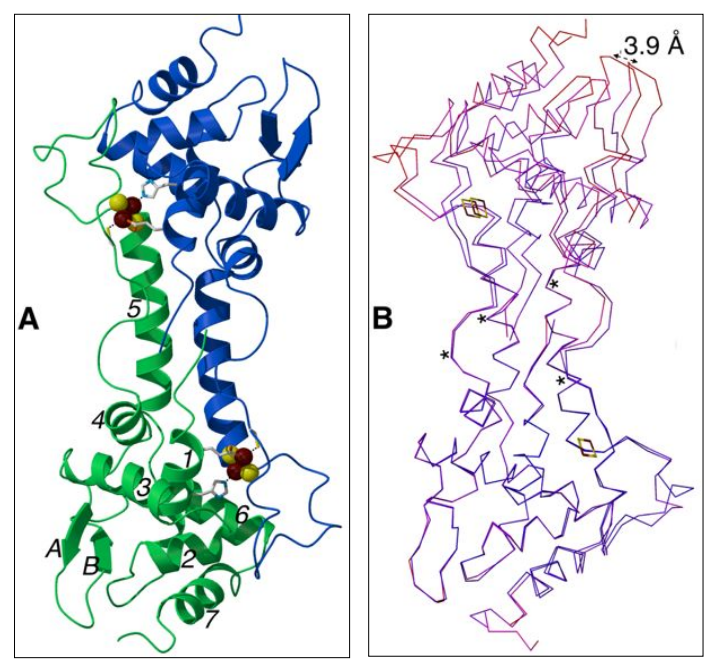

Figure 2. Structure of $S v$ RsrR in crystal RsrR-2 (Table S1). A. Ribbon representation of one dimer. Monomers are depicted in green and blue, respectively. [2Fe-2S] clusters are shown as brown $(\mathrm{Fe})$ and yellow $(\mathrm{S})$ spheres. Secondary structural elements are labeled 1-7 for $\alpha$-helices and A-B for $\beta$-strands. B. Comparison of the two $S v$ RsrR dimers in the asymmetric unit after superposition of one of their cluster binding domains. C $\alpha$ atom traces are colored from blue to red according to increasing temperature factors from 18 to $72 \AA^{2}$. Fe-S bonds are shown as thin sticks. Hinge regions near the center of the dimer are marked by asterisks.

There are two roughly aligned $S v$ RsrR dimers in the crystal asymmetric unit, a. u. Each $S v$ RsrR dimer shows the elongated fold typical of the Rrf2 family of transcriptional regulators, comprising seven $\alpha$-helices and two anti-parallel $\beta$-strands per monomer (Figure 2A). The X-ray structure parameters reveal an increase in temperature factors in the unit cell $\mathbf{c}$ direction for 
both dimers of the a. u., reflecting a small but significant conformational flexibility at their central region and some packing anisotropy (Figure 2B). The polypeptide chain of the four $S v$ RsrR monomers was resolved from residues 1 to 166 for the A chain, 1 to 158 for the $\mathrm{B}$ chain and 1 to 160 for the $\mathrm{C}$ and D chains.

A comparison with available related structures in the Protein Data Bank revealed that, although the DNA-binding N-terminal domain is mostly conserved, its conformation is variable. In terms of similarity, the global cysteine regulator CymR from Bacillus subtilis ${ }^{16}$ is the most similar to the $S v R s r R$ functional dimer, with a root mean-square deviation of $2.5 \AA$ for 125 of a total of 129 superposed $\mathrm{C} \alpha$ atoms when monomers are compared. Like other Rrf2 superfamily members, the $S v$ RsrR dimer buries an extensive surface (Figure 2), in this case of about $2400 \AA^{2}$ per monomer. Putative DNA-binding regions in the two $S v$ RsrR monomers, which are equivalent to those of other members of the Rrf2 family, are discussed below.

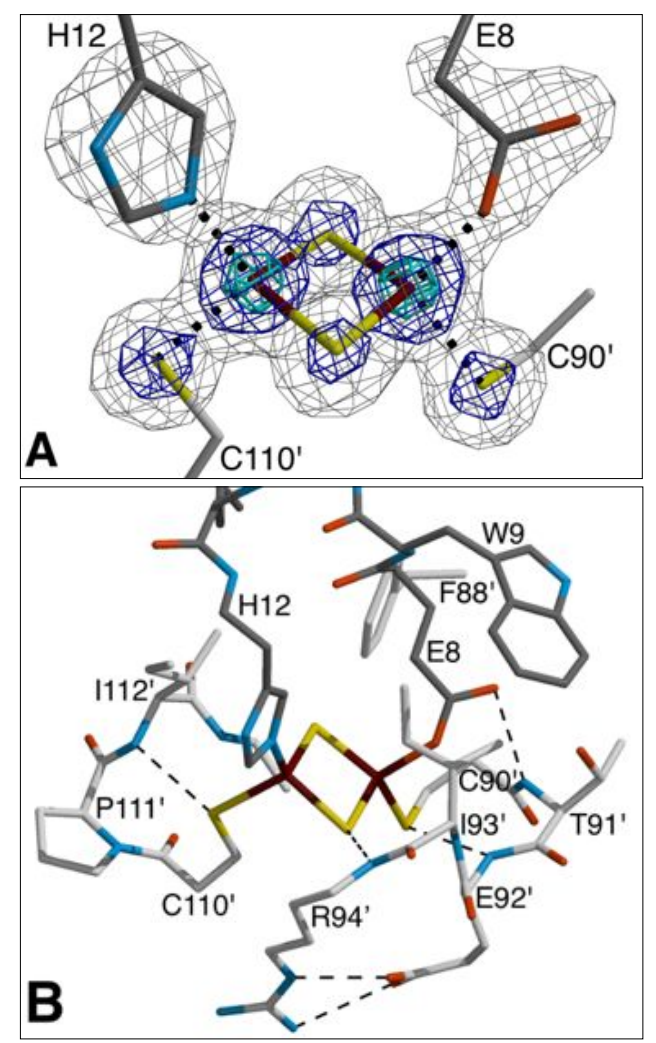

Figure 3. The [2Fe-2S] cluster of $\mathrm{S} v$ RsrR. A. Omit electron density map contoured at 5 (gray), 15 (blue) and 30 (cyan) times the r.m.s. level of the map. The positions of $\mathrm{Fe}$ and $\mathrm{S}$ atoms are clearly distinguished from those of lighter atoms. B. Environment of the cluster. Dashed lines indicate H-bonding interactions. Bonds between atoms are shown as sticks using the following atom colors: $\mathrm{Fe}$ brown, $\mathrm{S}$ yellow, $\mathrm{O}$ red, $\mathrm{N}$ blue and $\mathrm{C}$ dark-gray for one monomer and light-gray (and ' labels) for the other one. Trp9 is in its $\mathrm{W}_{\mathrm{o}}$ position (see text).

The dimeric holo-SvRsrR structure contains two [2Fe-2S] clusters that are ligated by Glu8 and His12 from one monomer and Cys90 and Cys110 from the other (Figure 3A). As mentioned above, $S v$ RsrR is the first structurally characterized protein with a $[2 \mathrm{Fe}-2 \mathrm{~S}]$ cluster, or any other kind of biological
[Fe-S] cluster, that is asymmetrically bound by three different types of protein ligands. Cys 90 is exposed to solvent, and stabilized by a long H-bond to the main chain $\mathrm{N}$ from Glu92, whereas the Cys $110 \mathrm{~S} \gamma$ atom is buried, mostly shielded by the side chains of Glu92 and Arg94, which form a salt bridge (Figure 3B). This $\mathrm{S} \gamma$ atom is stabilized by the positive dipole moment of the $\alpha 5$ helix. Otherwise, the cluster environment is mainly hydrophobic, involving its van der Waals interactions with the side chains of Phe88, Ile93, Ile112 and Ala113, and only one long $\mathrm{H}$-bond between one of its inorganic $\mathrm{S}$ atoms and the main chain $\mathrm{N}$ atom from Arg94. All the monomers have Trp9 in the already mentioned $\mathrm{W}_{\mathrm{o}}$ configuration, exposed edgeon to the solvent, with its indole heterocycle roughly parallel to the plane defined by the $[2 \mathrm{Fe}-2 \mathrm{~S}]$ rhomb and about $9.0 \AA$ from it (Figure 3B).

To investigate the importance of Glu8 and His12 for [2Fe-2S] cluster binding and redox properties, E8A, E8C, H12A, H12C and $\mathrm{E} 8 \mathrm{C} / \mathrm{H} 12 \mathrm{C}$ site-directed substituted variants of $\mathrm{SvRsrR}$ were generated and purified. In all cases, isolated proteins gave rise to absorption and $\mathrm{CD}$ spectra consistent with the presence of an [Fe-S] cluster (Figure 4). Mass spectrometry under nondenaturing conditions, which previously demonstrated the incorporation of a $[2 \mathrm{Fe}-2 \mathrm{~S}]$ cluster into wild type $S v \mathrm{RsrR},{ }^{9}$ confirmed the incorporation of a [2Fe-2S] cluster in each of these variant proteins (Figure S2 and Table S2). For E8A and H12A variants, the intensity of [2Fe-2S] cluster peaks was diminished, particularly in the dimer region, where only one cluster per $S v$ RsrR dimer was detected (Figures S2A and $\mathbf{C}$ ). On the contrary, replacement with Cys either had little effect on stability in the mass spectrometry experiment (E8C) or resulted in stabilization of the $[2 \mathrm{Fe}-2 \mathrm{~S}]$ cluster forms in both monomer and dimer regions (H12C). Stabilization was most apparent in the double Cys variant $\mathrm{E} 8 \mathrm{C} / \mathrm{H} 12 \mathrm{C}$, in which the cluster bound forms were by far the most abundant species (Figure S2E). Thus, the stability of the $S v \mathrm{RsrR}$ [2Fe-2S] cluster under the conditions of the mass spectrometry experiment varied depending on the cluster coordination. Substitution of a cluster ligand residue with a non-coordinating residue resulted in destabilization, whereas replacement with a Cys residue did not, and the putative all-Cys coordinated cluster was much more stable than the wild type cluster.

Comparison with the previously published absorption and CD (which is particularly sensitive to the cluster environment) spectra of wild type $S v R s r R$ in the reduced $(+1)$ and oxidized $(+2)$ state indicate some differences in the spectra of the Glu8 and His 12 variant proteins, consistent with changes in cluster coordination. ${ }^{9}$ In general the spectra more closely resemble that of the oxidized form of [2Fe-2S] RsrR, suggesting that the variant proteins were isolated with their cluster at least partially in the +2 oxidized state (Figure 4). This is in contrast to wild type $S v \mathrm{RsrR}$, which was isolated with its [2Fe-2S] cluster in the +1 state. ${ }^{9}$ The $[2 \mathrm{Fe}-2 \mathrm{~S}]$ cluster of wild type $S v R$ srR can cycle its oxidation state upon reaction with oxidant/reductant without affecting cluster stability. ${ }^{9}$ To determine the redox activity of the $[2 \mathrm{Fe}-2 \mathrm{~S}]$ cluster coordinated by the Glu8/His $12 \mathrm{SvRsrR}$ variants, protein samples were exposed to $\mathrm{O}_{2}$ to fully oxidize them and then were reduced with DTH (Figure S3). In all cases, exposure to $\mathrm{O}_{2}$ did not cause a change in the shape of the absorbance or CD spectra (Figure S3), consistent with the proposal that the variants were isolated with their clusters largely in an already oxidized, $[2 \mathrm{Fe}-2 \mathrm{~S}]^{2+}$, state. For each 
variant, addition of DTH resulted in a decrease in intensity in both absorbance and CD spectra, with no sign of signals characteristic of a reduced form of the cluster, indicating its loss. Consistent with this, samples that were re-exposed to $\mathrm{O}_{2}$ did not give rise to the re-appearance of the original spectra. These data demonstrate that, in contrast to the wild type protein, the $[2 \mathrm{Fe}-2 \mathrm{~S}]$ cluster in each of the $\mathrm{E} 8 \mathrm{~A} / \mathrm{H} 12 \mathrm{~A}$ variant proteins is unstable to redox cycling.
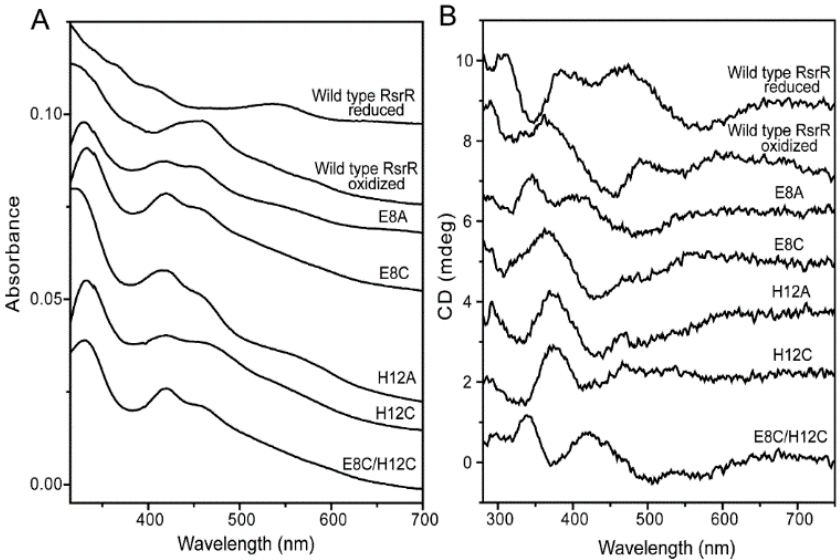

Figure 4. Spectroscopic characterization of Glu8/His12 variants of SvRsrR. UV-visible absorption (A) and CD (B) spectra of as isolated $\mathrm{E} 8 \mathrm{~A}, \mathrm{E} 8 \mathrm{C}, \mathrm{H} 12 \mathrm{~A}, \mathrm{H} 12 \mathrm{C}$ and $\mathrm{E} 8 \mathrm{C} / \mathrm{H} 12 \mathrm{C}$ SvRsrR variants, as indicated. Spectra were measured using a $1 \mathrm{~mm}$ sealed anaerobic cuvette. Absorbance and CD spectra of oxidized and reduced wild type $S \nu$ RsrR are shown for comparison. Proteins ( $80 \mu \mathrm{M}$ [2Fe-2S]) were in $50 \mathrm{mM}$ Tris, $2 \mathrm{M} \mathrm{NaCl}, 5 \%$ (v/v) glycerol, $\mathrm{pH} 8$.

The capacity of $S v$ RsrR variants to bind DNA containing an RsrR-regulated sequence was investigated by EMSA. None of the Glu8 or His12 variants exhibited significant specific DNAbinding (Figure 5). E8A exhibited a small amount of binding but this did not increase with increasing ratios of $S v$ RsrR and so this most likely does not represent bona fide reversible binding. A super-shifted band that was assigned to non-specific binding of the wild type protein was observed for all the variants (and was more apparent in both Glu8 single variants) with the exception of $\mathrm{H} 12 \mathrm{C}$, which did not exhibit binding of any kind. These data show that both Glu8 and His 12 are required for the cluster-bound protein to adopt the correct conformation for specific DNA-binding.

\section{Crystal Structure of an alternative RsrR conformation with differences in the DNA-binding region}

A crystal anaerobically grown from a methyl pentanediol and DTH-containing solution, and without reductant in the reservoir solution (see Methods), was flash-cooled inside the glove box and used to collect X-ray data to $2.0 \AA$ resolution (orthorhombic RsrR-3 in Table S1). Although the initial as-prepared protein solution was pink, after five weeks the crystal used for X-ray data collection was light brown (Figure S1B), suggesting partial cluster oxidation. We have previously observed partial oxidation of other crystallizing proteins in the glove box after several weeks, ${ }^{17}$ which is probably due to the slow release of trapped $\mathrm{O}_{2}$ from the plastic crystallization plates.

Importantly, this crystal has four mixed $\mathrm{W}_{\mathrm{o}} / \mathrm{W}_{\mathrm{i}}$ dimers in its a. u. The presence of these mixed dimers distinguishes this structure from the air-exposed RsrR-2 structure where, as mentioned above, all monomers have Trp9 in the $\mathrm{W}_{\mathrm{o}}$ configuration (Figure 3B and 6A).

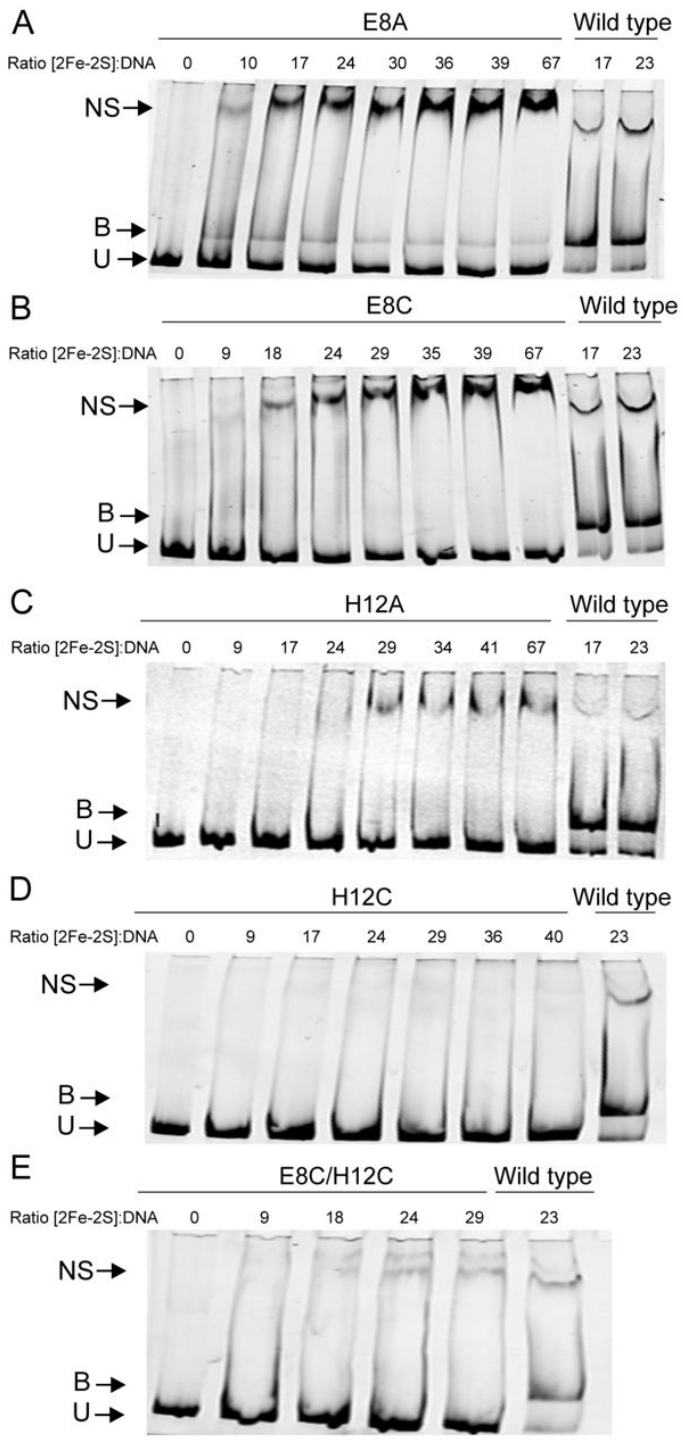

Figure 5. DNA-binding properties of $S v$ RsrR variant proteins with substitutions of cluster-coordinating residues. EMSAs showing RsrR-regulated DNA probes unbound, U, bound, B, and nonspecifically bound, NS by (A) E8A, (B) E8C, (C), H12A, (D) $\mathrm{H} 12 \mathrm{C}$, and (E) E8C/H12C SvRsrR. Ratios of [2Fe-2S] RsrR and [RsrR] to DNA are indicated. DNA concentration was $2.5 \mathrm{nM}$. The reaction mixtures were separated at $30 \mathrm{~mA}$ for $50 \mathrm{~min}$. Polyacrylamide gels were pre-run at $30 \mathrm{~mA}$ for $2 \mathrm{~min}$ prior to use.

The $\mathrm{W}_{\mathrm{o}} \rightarrow \mathrm{W}_{\mathrm{i}}$ conformational change involves a $96^{\circ}$ rotation about the $\mathrm{C} \alpha-\mathrm{C} \beta$ bond of the bulky Trp9 side chain into a preexisting protein cavity (Figure 6B). This rotation results in an overall indole displacement of about $9 \AA$ and maximal shifts of $6.2 \AA$ for Leu40, $2.8 \AA$ for Cys13 and $1.8 \AA$ for Tyr39. The rotated Trp9 interacts via a water molecule with the main chain $\mathrm{N}$ atom of His 33, which, in turn, shifts its imidazole group 3.9 $\AA$ towards the [2Fe-2S] cluster. The Trp9 rotation also disrupts an H-bonding network, which, in the $\mathrm{W}_{\mathrm{o}}$ conformation connected this residue via two ordered water molecules to the [2Fe-2S] cluster ligand Glu8 (Figure 6A). 
Overall, in its $\mathrm{W}_{\mathrm{i}}$ configuration Trp9 significantly modifies the positions of helices 2 and 3 of the RsrR DNA binding helixturn-helix (HTH) motif (see below and Figure 6C).

\section{Influence of crystal packing on RsrR conformation}

The identification of a second conformation of RsrR in crystals containing [2Fe-2S] RsrR in a partially reduced redox state (compared to that observed for the air-treated, oxidized form) suggested a link between conformation and oxidation state. To explore this further, a reduced form of [2Fe-2S] was investigated. Pink crystals (Figure S1C) anaerobically grown from a solution containing $2 \mathrm{mM}$ DTH and equilibrated against a reservoir solution with the same concentration of the reductant were obtained after four days (see Methods). X-ray data from one of these crystals were collected to $2.3 \AA$ resolution (RsrR4 in Table S1). The cell parameters of this monoclinic P2 crystal are very similar to those of the air-treated monoclinic crystal except for the $\mathrm{c}$ axis that is about $2 \AA$ shorter (Table S1). As in that structure, all dimers have Trp9 exposed to the solvent in the $\mathrm{W}_{\mathrm{o}}$ configuration.

The fact that i) both air-treated and fully reduced $\mathrm{P} 2{ }_{1}$ crystals have $\mathrm{W}_{\mathrm{o}} / \mathrm{W}_{\mathrm{o}}$ dimers and ii) the putatively partially reduced $\mathrm{P} 22_{1} 2_{1}$ crystal has $\mathrm{W}_{\mathrm{o}} / \mathrm{W}_{\mathrm{i}}$ dimers prompted us to evaluate the possible influence of crystal packing on Trp9 conformation. We found the $S v$ RsrR molecular contacts in the $\mathrm{P} 2{ }_{1}$ crystals incompatible with the $\mathrm{W}_{\mathrm{o}} / \mathrm{W}_{\mathrm{i}}$ arrangement in three out of the four RsrR dimers in the a. u. (Table S3).

The above analysis and the presence of $\mathrm{W}_{\mathrm{o}} / \mathrm{W}_{\mathrm{i}}$ dimers in the orthorhombic crystal, suggests that in a partially reduced $S v$ RsrR solution a substantial fraction of dimers is in this mixed $\mathrm{W}_{\mathrm{o}} / \mathrm{W}_{\mathrm{i}}$ conformational state. However, in our crystals the dimer composition seems to depend on the space group and the resulting molecular packing. Indeed, the observed rather subtle packing effect on the $\mathrm{W}_{\mathrm{o}} \rightarrow \mathrm{W}_{\mathrm{i}}$ conformational change of Trp9 is consistent with the fact that its two observed rotamers have the highest frequencies (lowest energy) in proteins: $36 \%$ for the exposed $\mathrm{W}_{\mathrm{o}}$ form and $18 \%$ for the buried $\mathrm{W}_{\mathrm{i}}$ species. ${ }^{18}$

\section{DNA-binding surface of holo-SvRsrR}

A straightforward superposition of $S v R s r R$ with IscR in complex with its cognate $\mathrm{DNA}^{3,4}$, besides indicating some differences in their interactions with the nucleic acid (Figure 7A), suggests that, in its $\mathrm{W}_{\mathrm{o}}$ configuration, the $\mathrm{N}$ indole atom of SvRsrR Trp9 could interact directly with the phosphate backbone of its DNA binding site (Figure 7B). This is also supported by the fact that IscR Ser5 and Lys6, which bind DNA, ${ }^{3,4}$ are replaced by Gly5 and Gly6 in $S v$ RsrR (Figure 1), thus possibly allowing for closer, main chain NH-OP interactions in the latter.
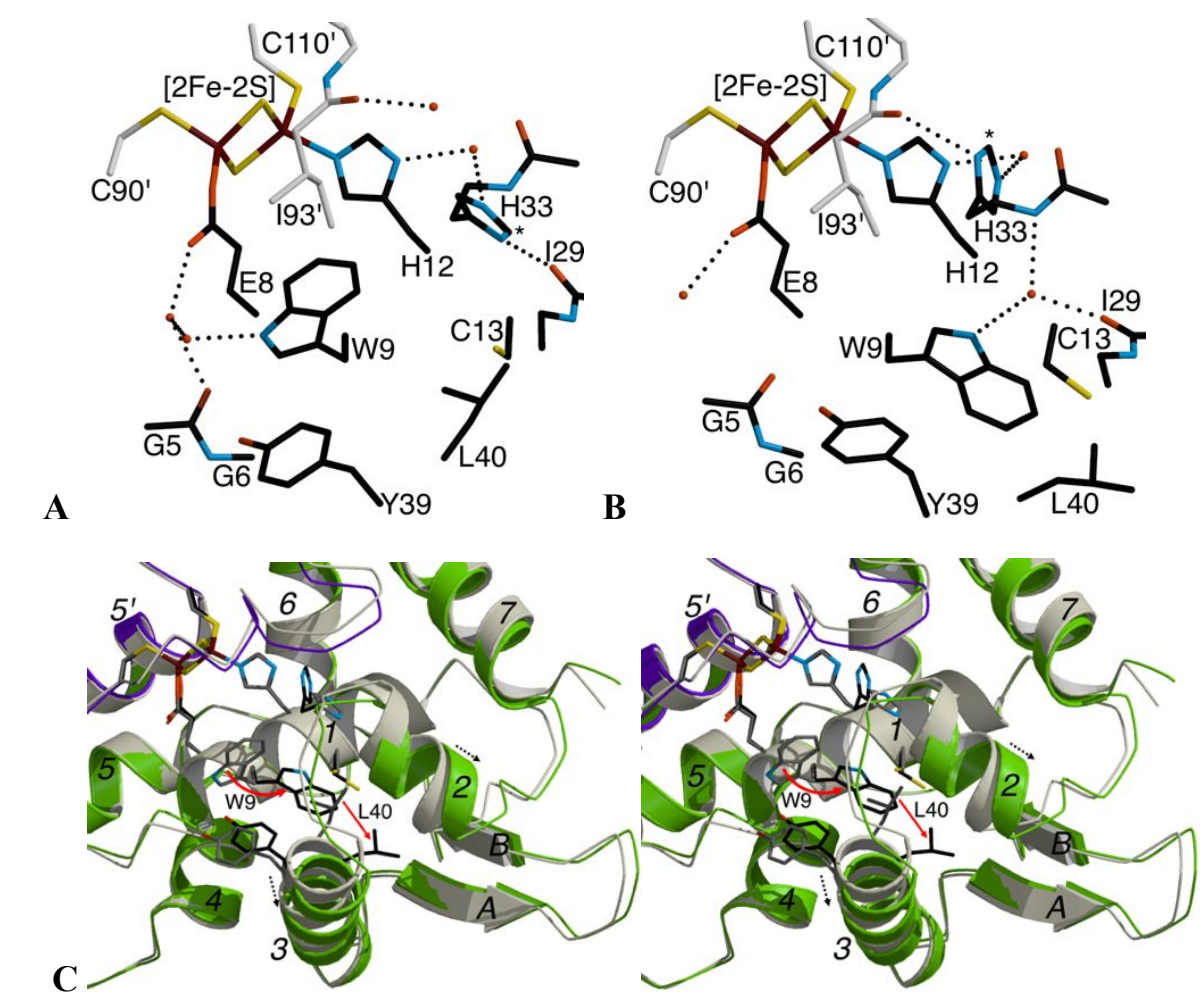

Figure 6. Conformational changes induced by the rotation of Trp9. A. Zoom of Trp9 and its environment in the $\mathrm{W}_{\mathrm{o}}$ configuration for crystal RsrR-2 (Table S1). B. The same region in the $\mathrm{W}_{\mathrm{i}}$ configuration for crystal RsrR-3 (see text and Table 1). The asterisk next to His 33 indicates the possible positively charged protonated state of this residue (based on its interactions). C. Stereo view of a superposition of the corresponding cluster-binding regions including the protein fold. Red arrows highlight the large movements of Trp9 and Leu40 and dashed black arrows indicate significant shifts of helices 2 and 3 of the DNA-binding helix-turn-helix motif. 

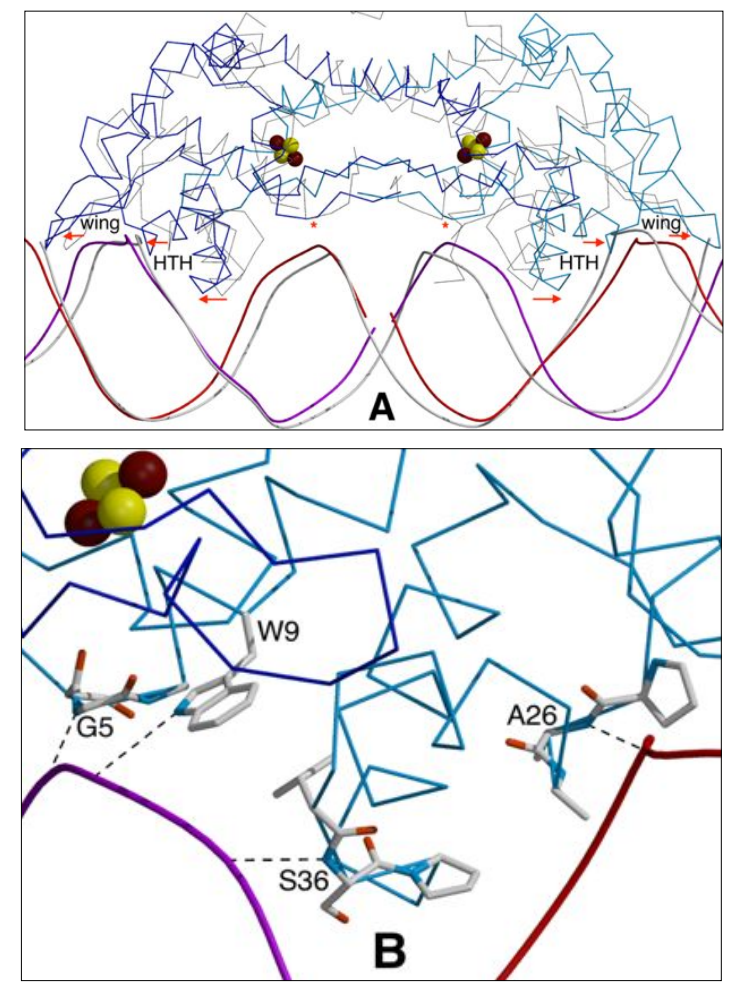

Figure 7. The DNA binding surface of $S v$ RsrR. A. Superposition of the IscR-DNA complex (gray, 29 base pairs) to one RsrR dimer (light- and dark-blue). The DNA binding HTH and wing motifs are significantly more separated in RsrR. When IscR and RsrR monomers are superimposed instead, the corresponding 15 base pairs DNA fragments (shown in red and violet) are shifted in the direction of the red arrows. B. Zoom on potential contacts with DNA for one RsrR monomer based on the superposition of half of the IscR-DNA complex (pdb code $4 \mathrm{hfl}$ ).

\section{DISCUSSION}

The crystal structures of $S v$ RsrR show an unprecedented coordination of its [2Fe-2S] cluster with three different types of protein ligands, His, Glu and Cys. The importance of Glu8 and His 12 has been demonstrated here mainly by the finding that the His $\rightarrow$ Cys and Glu $\rightarrow$ Cys substitutions, although still enabling coordination of a [2Fe-2S] cluster, result in impaired DNA binding and instability to redox cycling. Besides possibly modulating the cluster's reduction potential and properties, the unusual coordination may have a structural basis: The $\mathrm{C} \alpha$-to-Fe distance for Glu8 $(6.1 \AA)$ and for His12 $\AA(6.5 \AA)$ are significantly longer than the corresponding values for Cys 90 (3.8 $\AA)$ and Cys110 (4.5 $\AA$ ). This difference determines the positions of the N-terminal residues Gly5 and Gly6 and of Trp9 close to or at the DNA-binding site (Figure 7B). It may also explain why the $\mathrm{H} 12 \mathrm{C}$ and $\mathrm{E} 8 \mathrm{C}$ substitutions abolish the binding of $S v$ RsrR to the nucleic acid; shorter C $\alpha$-to-Fe distances would pull the N-terminal region towards the cluster, modifying the position of the corresponding residues.

$S v$ RsrR has the remarkable property of modulating its DNA binding through a one-electron redox process at its [2Fe-2S] cluster. ${ }^{9}$ Understanding such subtle control from a structural viewpoint poses a major challenge. Our structures revealed two RsrR conformations, $\mathrm{W}_{\mathrm{o}}$ and $\mathrm{W}_{\mathrm{i}}$, that suggest how conformational changes could modulate DNA-binding, and we now need to further investigate the $\mathrm{W}_{\mathrm{o}} \rightarrow \mathrm{W}_{\mathrm{i}}$ switch to establish if it is correlated with the redox state of the cluster, and thus whether it is the conformational change provoked by the reduction of the cluster in cellulo. We note that tryptophan is not an electrostatically-innocent amino acid. Indeed, in its ground state its indole heterocycle has a dipole moment $\mathbf{d}$ of about 2-3 Debye. ${ }^{19}$ In addition, as mentioned above, the burial of Trp9 modifies the conformation of several regions, especially the Pro25-Leu40 segment from the HTH motif (Figure 6C), which binds to its cognate DNA major groove.

We also note that the N-terminal EWxxH motif of $S v$ RsrR is found in several other predicted Rrf2 family regulators from many bacterial phyla, suggesting that the unusual iron-sulfur cluster coordination and possible functional importance of Trp9 could be widespread (Figure S4).

The work described here highlights the remarkable mechanistic variety that exists within the Rrf2 family of transcriptional regulators, which includes, in addition to RsrR, the iron-sulfur cluster biogenesis regulator $\mathrm{IscR}^{2-4,6}$, the nitric oxide sensor $\mathrm{NsrR}^{5}$ and the iron regulator RirA. ${ }^{8}$ Structures of RsrR, IscR and NsrR reveal a well conserved overall fold, but the nature of the cluster and its coordination in each case is distinct. The structure of [4Fe-4S] RirA is not yet available but none of the non-Cys coordinating residues of RsrR, NsrR or IscR are conserved in the RirA sequence. The observed variation, while undoubtedly reflecting the different signals that are sensed by each regulator, raises the question of how the protein framework accommodates such plasticity at the cluster and its environment.

In summary, we have determined the crystal structure of $S v$ RsrR, a new member of the Rrf2 superfamily of dimeric bacterial transcription factors, and found it to have a novel type of [2Fe-2S] cluster coordinated by one Glu and one His from one monomer and two Cys residues from the other. We have also shown that this coordination is essential for DNA binding and redox cycling. Furthermore, it appears that Trp9 rotation might modulate the $S v$ RsrR binding affinity for the nucleic acid. Next, we will further explore the functional role of this residue using site-directed mutagenesis and fluorescence spectroscopy.

\section{METHODS}

Purification of wild type Streptomyces venezuelae RsrR and variant proteins. C-terminally His-tagged wild type $S v$ RsrR was purified as previously described. ${ }^{9}$ Plasmids for the purification of E8A, E8C, H12A, H12C, and E8C/H12C sitedirected variants of C-terminally His-tagged $\mathrm{SvRsrR}$ were purchased from Genscript. Variant proteins were purified using the same procedure as for wild type $S v$ RsrR. Briefly, E. coli BL21 (DE3) containing the appropriate plasmid was grown in Luria-Bertani medium supplemented with $20 \mu \mathrm{M}$ ammonium ferric citrate at $37{ }^{\circ} \mathrm{C}, 200 \mathrm{rpm}$ until $\mathrm{OD}_{600 \mathrm{~nm}}$ was 0.6-0.9. Cultures were cold shocked (18 $\mathrm{min}$ on ice) prior to induction (100 $\mu \mathrm{M}$ IPTG), further supplementation with $200 \mu \mathrm{M}$ ammonium ferric citrate and $25 \mu \mathrm{M}$ L-methionine, and incubation at $30{ }^{\circ} \mathrm{C}$ and $105 \mathrm{rpm}$ and incubated for a further 3.5 $\mathrm{h}$ at $30{ }^{\circ} \mathrm{C}$. Cells were lysed by sonication and the resulting supernatant applied anaerobically to a HiTrap IMAC HP (GE Healthcare) column equilibrated in $50 \mathrm{mM}$ Tris, $100 \mathrm{mM} \mathrm{CaCl}_{2}$, $200 \mathrm{mM}$ L-cysteine, 5\% (v/v) glycerol, $\mathrm{pH} 8$, and eluted using a linear gradient from 0 to $100 \% 200 \mathrm{mM}$ histidine in the same buffer. A HiTrap Heparin (1 x 1mL; GE Healthcare) column under anaerobic conditions was used to remove the cysteine and 
histidine, using $50 \mathrm{mM}$ Tris, $2 \mathrm{M} \mathrm{NaCl}, 5 \%$ (v/v) glycerol, $\mathrm{pH} 8$ to elute the protein. $S v$ RsrR protein concentrations were determined using the method of Bradford (Bio-Rad Laboratories), ${ }^{20}$ with $\mathrm{BSA}$ as the standard. Cluster concentrations were determined using $\varepsilon_{455 \mathrm{~nm}}=3450 \pm 25 \mathrm{M}^{-1} \mathrm{~cm}^{-}$ ${ }^{1}$ for the oxidized form, ${ }^{9}$ while those for variant proteins were determined via Ferene iron determination assays, as previously described. $^{21}$ Cluster loadings were as follows: Wild type RsrR, $83 \%$; E8A, 53\%; E8C, 73\%; H12A, 27\%; H12C, 77\%; E8C/H12C, 92\%. RsrR [2Fe-2S] clusters were oxidized by exposure to atmospheric $\mathrm{O}_{2}$ and incubation for $10-30 \mathrm{~min}$. Kinetic experiments showed that oxidation occurred rapidly $(<$ $1 \mathrm{~min}$ ). Reduction was achieved through the addition of either a 1:1 ratio of sodium DTH (where multiple redox cycling was performed), or a 4-fold excess of DTH. Reduction occurred immediately though, in some cases, samples were incubated prior to spectral measurement. For the wild type protein the oxidized and reduced forms were entirely stable and could be redox cycled numerous times without significant cluster loss or protein precipitation.

Spectroscopy and mass spectrometry. UV-visible absorbance measurements were performed using a Jasco V500 spectrometer, and CD spectra were measured with a Jasco J810 spectropolarimeter. For mass spectrometry under nondenaturing conditions, $S v$ RsrR proteins were exchanged into $250 \mathrm{mM}$ ammonium acetate, $\mathrm{pH}$ 8, using PD10 desalting columns (GE Life Sciences), diluted to $\sim 21 \mu \mathrm{M}$ cluster and infused directly $(0.3 \mathrm{~mL} / \mathrm{h})$ into the ESI source of a Bruker micrOTOF-QIII mass spectrometer (Bruker Daltonics, Coventry, UK) operating in the positive ion mode. Data were acquired over the $\mathrm{m} / \mathrm{z}$ range $700-3500$, with the following parameters: dry gas flow $4 \mathrm{~L} / \mathrm{min}$, nebuliser gas pressure 0.8 Bar, dry gas $180{ }^{\circ} \mathrm{C}$, capillary voltage $2750 \mathrm{~V}$, offset $500 \mathrm{~V}$, ion energy $5 \mathrm{eV}$, collision RF $180 \mathrm{Vpp}$, collision cell energy 10 eV. ${ }^{22}$ The ESI-TOF was calibrated using ESI-L low concentration tuning mix (Agilent Technologies, San Diego, CA). Prior to the introduction of samples, the gas tight syringe (Hamilton) and associated PEEK tubing (Upchurch Scientific) were flushed with $5 \mathrm{ml}$ of anaerobic ammonium acetate buffer. Processing and analysis of MS experimental data was carried out using Compass DataAnalysis version 4.1 (Bruker Daltonik, Bremen, Germany). Neutral mass spectra were generated using the ESI Compass version 1.3 Maximum Entropy deconvolution algorithm. Exact masses are reported from peak centroids representing the isotope average neutral mass. For apo-proteins, these are derived from $\mathrm{m} / \mathrm{z}$ spectra, for which peaks correspond to $[\mathrm{M}+\mathrm{nH}] \mathrm{n}+/ \mathrm{n}$. For cluster- or cluster derivative-containing proteins, where the cluster contributes charge, peaks correspond to $\left[\mathrm{M}+\mathrm{FeS}^{\mathrm{x}^{+}}+(\mathrm{n}-\mathrm{x}) \mathrm{H}\right]^{\mathrm{n}+} / \mathrm{n}$, where $\mathrm{M}$ is the molecular mass of the protein, $\mathrm{FeS}$ is the mass of the iron-sulfur cluster of $\mathrm{x}^{+}$ charge, $\mathrm{H}$ is the mass of the proton and $\mathrm{n}$ is the total charge. In the expression, the $\mathrm{x}+$ charge of the cluster offsets the number of protons required to achieve the observed charge state $(n+) .{ }^{23}$ Predicted masses are given as the isotope average of the neutral protein or protein complex, in which cofactor-binding is expected to be charge compensated. ${ }^{23-25}$

Electrophoretic mobility shift assays (EMSAs). Bandshift reactions $(20 \mu \mathrm{l})$ were carried out on ice in $10 \mathrm{mM}$ Tris, $60 \mathrm{mM}$ $\mathrm{KCl}$, pH 7.52 using the 5' 6-FAM-labelled intergenic region between $S$. venezualae sven 1847 and sven 1848 , as previously described. ${ }^{9}$ Briefly, $1 \mu \mathrm{L}$ of DNA was titrated with varying aliquots of variant or wild type $S v$ RsrR proteins. $2 \mu \mathrm{L}$ of loading dye (containing $0.01 \%$ (w/v) bromophenol blue), was added and the reaction mixtures were immediately separated at $30 \mathrm{~mA}$ on a $5 \%(\mathrm{w} / \mathrm{v})$ polyacrylamide gel in $1 \mathrm{x}$ TBE $(89 \mathrm{mM}$ Tris, $89 \mathrm{mM}$ boric acid, 2 mM EDTA), using a Mini Protean III system (BioRad). Gels were visualized (excitation, $488 \mathrm{~nm}$; emission, 530 $\mathrm{nm}$ ) on a molecular imager FX Pro (Bio-Rad). Polyacrylamide gels were pre-run at $30 \mathrm{~mA}$ for $2 \mathrm{~min}$ prior to use.

Crystallization and $\mathrm{X}$-ray structure determination of the different $S v$ RsrR structures.

As-isolated wild-type $\boldsymbol{S} \boldsymbol{v} \mathbf{R s r} \mathrm{R}$. Initial crystallization trials were carried out by screening 1248 conditions from 13 different commercial kits by the vapor diffusion method with a Gryphon robot (Art Robin Instruments, $C A, U S A$ ) in an anaerobic glove box. Each sitting drop was prepared by mixing $200 \mathrm{~nL}$ of a 20 $\mathrm{mg} / \mathrm{mL}$ of as-isolated $\mathrm{SvRsrR}$ in $300 \mathrm{mM} \mathrm{NaCl}, 5 \%(\mathrm{v} / \mathrm{v})$ glycerol, $50 \mathrm{mM}$ Tris Base $\mathrm{pH} 8.0$ with $200 \mathrm{~nL}$ of the commercial crystallization solution. The drop was then equilibrated against $100 \mu \mathrm{L}$ of the latter at $20^{\circ} \mathrm{C}$. Useful crystals were obtained from two conditions. In one of them the commercial crystallization solution was composed of $20 \%$ PEG6000 and $100 \mathrm{mM}$ MES $\mathrm{pH}$ 6.0. This crystallization experiment was scaled up manually and the best crystals were obtained by mixing $1 \mu \mathrm{L}$ of the protein-containing solution described above with $1 \mu \mathrm{L}$ of a 27\% PEG6000, $100 \mathrm{mM}$ MES, $\mathrm{pH} 6.5$ crystallization solution, equilibrated against $1 \mathrm{~mL}$ of the latter at $20{ }^{\circ} \mathrm{C}$. A crystal obtained under these conditions after one week was then transferred to a cryo-protecting solution composed of $40 \%$ PEG6000, 20\% glycerol and $100 \mathrm{mM}$ MES $\mathrm{pH}$ 6.5, mounted in a cryo-loop and flash-cooled in liquid propane ${ }^{26}$ inside the glove box. This crystal, which belonged to space group $\mathrm{P} 2{ }_{1}$, was used for the initial structure determination (see RsrR-1 in Table S1).

Air-treated wild-type $\boldsymbol{S v} \mathbf{R s r R}$. A second crystal from the same drop was taken out of the glove box and exposed to air for 10 min in oxygenated cryo-protecting solution without DTH before being mounted and flash-cooled with liquid propane. This crystal, which also belonged to space group $\mathrm{P} 2{ }_{1}$, was used to collect X-ray data to $1.6 \AA$ resolution and was considered to correspond to oxidized $S v R s r R$ (see RsrR-2 in Table S1).

Partially-reduced wild-type -SvRsrR. In the second condition from the screening that gave crystals the commercial solution was composed of $65 \%$ methyl-pentanediol, $100 \mathrm{mM} \mathrm{MES}, \mathrm{pH}$ 6.0. In this experiment $2 \mathrm{mM}$ DTH was only included in the protein drop. After five weeks, one of these crystals, mounted directly from the plate set up by the robot, was used to collect $\mathrm{X}$-ray data to $2.0 \AA$ resolution and was found to belong to the orthorhombic space group P $2{ }_{1} 2_{1} 2_{1}$ (see RsrR-3 in Table S1).

Dithionite-reduced wild-type SvRsrR. A new series of crystals were obtained from a second protein batch using the same strategy except that $1 \mathrm{~mL}$ of a $100 \mu \mathrm{M}$ DTH, $10 \mathrm{mM}$ $\mathrm{NaOH}$ solution, previously sparged with the glove box atmosphere, was added to $50 \mu \mathrm{L}$ of the $S v$ RsrR-containing solution described above. Crystallization drops were prepared as described above except that the crystallization solution was 14\% PEG6000, 2 mM DTH, $100 \mathrm{mM}$ MES, pH 6.5. The protein sample used in this experiment remained pink upon thawing and a freshly prepared DTH solution was used. A crystal grown under these conditions was found to belong to space group $\mathrm{P} 2$ (RsrR-4 in Table S1). The crystal appeared after two days and 
was subsequently transferred to the cryo-protecting solution with added $2 \mathrm{mM}$ DTH, mounted in a cryo-loop and flashcooled in liquid propane inside the glove box. X-ray diffraction data were collected to $2.3 \AA$ resolution.

$\mathrm{X}$-ray structure determination. All X-ray diffraction data were collected at the European Synchroton Radiation Facility in Grenoble with $\mathrm{MXCuBe},{ }^{27}$ using a Pilatus $6 \mathrm{M}$ (Dectris) detector on beamlines ID23-1 and ID30B, a Pilatus X 2M (Dectris) detector on beamline ID23-2 and an EIGER X 4M (Dectris) detector on beamline ID30-A3 (Table S1). The data were indexed, integrated and scaled with $X D S^{28}$ and submitted to a final scaling and merging step with AIMLESS. $^{29}$ An X-ray absorption spectrum was measured for crystal RsrR-1 on a Rontec Xflash Xray fluorescence detector and treated with $\mathrm{CHOOCH}^{30}$ to find the wavelength for maximal anomalous scattering near the $\mathrm{Fe}$ absorption edge. A highly redundant data set was obtained at this wavelength by combining two $360^{\circ}$ data collections from the same crystal, centered at different positions. A subsequent Hybrid Substructure Search with PHENIX ${ }^{31}$ located $8 \mathrm{Fe}$ metal sites, which corresponded to 4 [2Fe-2S]

\section{ASSOCIATED CONTENT}

\section{Supporting Information}

The Supporting Information is available free of charge on the ACS Publications website.

$x$-ray crystallographic and mass spectrometric details and supporting figures (PDF).

\section{AUTHOR INFORMATION}

\section{Corresponding Author}

* Correspondence should be addressed to either N.Lebrun@uea.ac.uk or juan.fontecilla@ibs.fr.

\section{ORCID}

Nick Le Brun: 0000-0001-9780-4061

Juan C. Fontecilla-Camps: 0000-0002-3901-1378

Anne Volbeda: 0000-0002-6038-0979

\section{Present Addresses}

\$ Centro de Biotecnología y Genómica de Plantas, Universidad Politécnica de Madrid, Instituto Nacional de Investigación y Tecnología Agraria y Alimentaria, Pozuelo de Alarcón, 28223 Madrid, Spain

\section{Author Contributions}

The manuscript was written through contributions of all authors. All authors have given approval to the final version of the manuscript.

\section{Funding Sources}

This work was partially supported by FRISBI (ANR-10-INSB-0502) within the Grenoble Partnership for Structural Biology (PSB). This work was also supported by the Biotechnology and Biological Sciences Research Council through grants BB/J003247/1, BB/L007673/1 and BB/P006140/1, by the Natural Environment Research Council via the award of a PhD studentship to JTM, by UEA through the award of a PhD studentship to MTPM and purchase of the ESI-MS instrument, and by the FeSBioNet COST Action CA15133. clusters per asymmetric unit. A good starting model of RsrR was next obtained using the automated experimental phasing and model building (AutoSol) routines of PHENIX. Manual model corrections were performed with $C O O T^{32}$ and atomic positions and anisotropic temperature factors were refined with REFMAC $5^{33}$ using the $1.60 \AA$ resolution diffraction data collected for crystal RsrR-2. The resulting atomic coordinates were used as a starting model to solve the structures of the wild type crystals RsrR-3 and RsrR-4 with PHASER.${ }^{34}$ Compared to the starting monoclinic form a different crystal form was obtained for RsrR-3, whereas the monoclinic crystal RsrR-4 displayed significantly different cell dimensions. For these other crystals, REFMAC5 was first used for rigid body refinement and next for refinement of atomic positions and isotropic temperature factors, refining also TLS parameters that were defined for the individual RsrR subunits. Final refinement statistics are given in Table S1. The protein data bank ID codes for the deposited atomic coordinates and structure factors are 6HSD (RsrR-2), 6HSM (RsrR-3) and 6HSE (RsrR-4).

\section{Notes}

The authors declare no competing financial interests.

\section{ACKNOWLEDGMENT}

The Metalloproteins Unit members thank the CEA, the CNRS and the Université Grenoble-Alpes for institutional funding and the beamlines ID30-A3, ID30B, ID23-1 and ID23-2 of the European Synchrotron Radiation Facility (ESRF) for help with X-ray data collection. This work used the platforms of the Grenoble Instruct center (ISBG; UMS 3518 CNRS-CEA-UJF-EMBL) with support from FRISBI (ANR-10-INSB-05-02) and GRAL (ANR-10-LABX49-01) within the Grenoble Partnership for Structural Biology (PSB). We also thank Imen Khtatfi for help with the experiments.

\section{ABBREVIATIONS}

6-FAM, 6-Carboxyfluorescein; EMSA, electrophoretic mobility shift assay; PEEK, polyetheretherketone

\section{REFERENCES}

(1) Hibbing, M. E.; Fuqua, C. Antiparallel and Interlinked Control of Cellular Iron Levels by the Irr and RirA Regulators of Agrobacterium tumefaciens. J. Bacteriol. 2011, 193, 3461-3472.

(2) Santos, J. A.; Pereira, P. J.; Macedo-Ribeiro, S. What a Difference a Cluster Makes: The Multifaceted Roles of IscR in Gene Regulation and DNA Recognition. Biochim. Biophys. Acta 2015, 1854, 1101-1112.

(3) Santos, J. A.; Alonso-Garcia, N.; Macedo-Ribeiro, S.; Pereira, P. J. The Unique Regulation of Iron-Sulfur Cluster Biogenesis in a Gram-Positive Bacterium. Proc. Natl. Acad. Sci. U. S. A. 2014, 111, E2251-E2260.

(4) Rajagopalan, S.; Teter, S. J.; Zwart, P. H.; Brennan, R. G.; Phillips, K. J.; Kiley, P. J. Studies of IscR Reveal a Unique Mechanism for Metal-Dependent Regulation of DNA Binding Specificity. Nat. Struct. Mol. Biol. 2013, 20, 740-747.

(5) Volbeda, A.; Dodd, E. L.; Darnault, C.; Crack, J. C.; Renoux, O.; Hutchings, M. I.; Le Brun, N. E.; Fontecilla-Camps, J. C. Crystal Structures of the NO Sensor NsrR Reveal How Its Iron-Sulfur Cluster Modulates DNA Binding. Nat. Commun. 2017, 8, 15052.

(6) Yeo, W. S.; Lee, J. H.; Lee, K. C.; Roe, J. H. IscR Acts as an Activator in Response to Oxidative Stress for the Suf Operon Encoding Fe-S Assembly Proteins. Mol. Microbiol. 2006, 61, 206-218.

(7) Nesbit, A. D.; Giel, J. L.; Rose, J. C.; Kiley, P. J. SequenceSpecific Binding to a Subset of IscR-Regulated Promoters Does Not Require IscR Fe-S Cluster Ligation. J. Mol. Biol. 2009, 387, $28-41$. 
(8) Bhubhanil, S.; Niamyim, P.; Sukchawalit, R.; Mongkolsuk, S. Cysteine Desulphurase-Encoding Gene SufS2 Is Required for the Repressor Function of RirA and Oxidative Resistance in Agrobacterium tumefaciens. Microbiol. 2014, 160, 79-90.

(9) Munnoch, J. T.; Martinez, M. T.; Svistunenko, D. A.; Crack, J. C.; Le Brun, N. E.; Hutchings, M. I. Characterization of a Putative NsrR Homologue in Streptomyces venezuelae Reveals a New Member of the Rrf2 Superfamily. Sci. Rep. 2016, 6, 31597.

(10) Newton, G. L.; Buchmeier, N.; Fahey, R. C. Biosynthesis and Functions of Mycothiol, the Unique Protective Thiol of Actinobacteria. Microbiol. Mol. Biol. Rev. 2008, 72, 471-494.

(11) Kobayashi, K. Sensing Mechanisms in the Redox-Regulated, [2Fe-2S] Cluster-Containing, Bacterial Transcriptional Factor SoxR. Acc. Chem. Res. 2017, 50, 1672-1678.

(12) Fujikawa, M.; Kobayashi, K.; Kozawa, T. Redox-Dependent DNA Distortion in a SoxR Protein-Promoter Complex Studied Using Fluorescent Probes. J. Biochem. 2015, 157, 389-397.

(13) Bradley, T. M.; Hidalgo, E.; Leautaud, V.; Ding, H.; Demple, B. Cysteine-to-Alanine Replacements in the Escherichia coli SoxR Protein and the Role of the $[2 \mathrm{Fe}-2 \mathrm{~S}]$ Centers in Transcriptional Activation. Nucleic Acids Res. 1997, 25, 1469-1475.

(14) Holm, L.; Laakso, L. M. Dali Server Update. Nucleic Acids Res. 2016, 44, W351-355.

(15) Kabsch, W.; Sander, C. Dictionary of Protein Secondary Structure: Pattern Recognition of Hydrogen-Bonded and Geometrical Features. Biopolymers 1983, 22, 2577-2637.

(16) Shepard, W.; Soutourina, O.; Courtois, E.; England, P.; Haouz, A.; Martin-Verstraete, I. Insights into the Rrf2 Repressor Family--the Structure of CymR, the Global Cysteine Regulator of Bacillus subtilis. FEBS J 2011, 278, 2689-2701.

(17) Nicolet, Y.; Rohac, R.; Martin, L.; Fontecilla-Camps, J. C. X-Ray Snapshots of Possible Intermediates in the Time Course of Synthesis and Degradation of Protein-Bound $\mathrm{Fe}_{4} \mathrm{~S}_{4}$ Clusters. Proc. Natl. Acad. Sci. U. S. A. 2013, 110, 7188-7192.

(18) Hintze, B. J.; Lewis, S. M.; Richardson, J. S.; Richardson, D. C. MolProbity's Ultimate Rotamer-Library Distributions for Model Validation. Proteins 2016, 84, 1177-1189.

(19) Vivian, J. T.; Callis, P. R. Mechanisms of Tryptophan Fluorescence Shifts in Proteins. Biophys. J. 2001, 80, 2093-2109.

(20) Bradford, M. M. A Rapid and Sensitive Method for the Quantitation of Microgram Quantities of Protein Utilizing the Principle of Protein-Dye Binding. Anal. Biochem. 1976, 72, 248-254.

(21) Crack, J. C.; Gaskell, A. A.; Green, J.; Cheesman, M. R.; Le Brun, N. E.; Thomson, A. Influence of the environment on the [4Fe$4 \mathrm{~S}] 2+$ to $[2 \mathrm{Fe}-2 \mathrm{~S}] 2+$ cluster switch in the transcriptional regulator FNR. J. Am. Chem. Soc. 2008, 130, 1749-1758

(22) Laganowsky, A.; Reading, E.; Hopper, J. T. S.; Robinson, C. V. Mass Spectrometry of Intact Membrane Protein Complexes. Nat. Protoc. 2013, 8, 639-651.
(23) Johnson, K. A.; Verhagen, M. F.; Brereton, P. S.; Adams, M. W.; Amster, I. J. Probing the Stoichiometry and Oxidation States of Metal Centers in Iron-Sulfur Proteins Using Electrospray FTICR Mass Spectrometry. Anal Chem 2000, 72, 1410-1418.

(24) Kay, K. L.; Hamilton, C. J.; Le Brun, N. E. Mass Spectrometry of $B$. subtilis CopZ: $\mathrm{Cu}(\mathrm{i})$-Binding and Interactions with Bacillithiol. Metallomics 2016, 8, 709-719.

(25) Crack, J. C.; Thomson, A. J.; Le Brun, N. E. Mass Spectrometric Identification of Intermediates in the $\mathrm{O}_{2}$-Driven [4Fe-4S] to [2Fe-2S] Cluster Conversion in FNR. Proc. Natl. Acad. Sci. U. S. A. 2017, 114, E3215-E3223.

(26) Vernède, X.; Fontecilla-Camps, J. C. A Method to Stabilize Reduced and/or Gas-Treated Protein Crystals by Flash-Cooling under a Controlled Atmosphere. J. Appl. Cryst. 1999, 32, 505-509.

(27) Gabadinho, J.; Beteva, A.; Guijarro, M.; Rey-Bakaikoa, V.; Spruce, D.; Bowler, M. W.; Brockhauser, S.; Flot, D.; Gordon, E. J.; Hall, D. R.; Lavault, B.; McCarthy, A. A.; McCarthy, J.; Mitchell, E.; Monaco, S.; Mueller-Dieckmann, C.; Nurizzo, D.; Ravelli, R. B. G.; Thibault, X.; Walsh, M. A.; Leonard, G. A.; McSweeney, S. M. MxCuBE: A Synchrotron Beamline Control Environment Customized for Macromolecular Crystallography Experiments. J. Synchrotron Radiat. 2010, 17, 700-707.

(28) Kabsch, W. XDS. Acta Crystallogr. D Biol. Crystallogr. 2010, 66, 125-132.

(29) Evans, P. R.; Murshudov, G. N. How Good Are My Data and What Is the Resolution? Acta Crystallogr. D Biol. Crystallogr. 2013, 69, 1204-1214.

(30) Evans, G.; Pettifer, R. F. CHOOCH: A Program for Deriving Anomalous-Scattering Factors from X-Ray Fluorescence Spectra. J. Appl. Crystallogr. 2001, 34, 82-86.

(31) Adams, P. D.; Afonine, P. V.; Bunkóczi, G.; Chen, V. B.; Davis, I. W.; Echols, N.; Headd, J. J.; Hung, L.-W.; Kapral, G. J.; Grosse-Kunstleve, R. W.; McCoy, A. J.; Moriarty, N. W.; Oeffner, R.; Read, R. J.; Richardson, D. C.; Richardson, J. S.; Terwilliger, T. C.; Zwart, P. H. PHENIX: A Comprehensive Python-Based System for Macromolecular Structure Solution. Acta Crystallogr. D Biol. Crystallogr. 2010, 66, 213-221.

(32) Emsley, P.; Lohkamp, B.; Scott, W. G.; Cowtan, K. Features and Development of Coot. Acta Crystallogr. D Biol. Crystallogr. 2010, $66,486-501$.

(33) Murshudov, G. N.; Skubák, P.; Lebedev, A. A.; Pannu, N. S.; Steiner, R. A.; Nicholls, R. A.; Winn, M. D.; Long, F.; Vagin, A. A. REFMAC5 for the Refinement of Macromolecular Crystal Structures. Acta Crystallogr. D Biol. Crystallogr. 2011, 67, 355-367.

(34) McCoy, A. J.; Grosse-Kunstleve, R. W.; Adams, P. D.; Winn, M. D.; Storoni, L. C.; Read, R. J. Phaser Crystallographic Software. J. Appl. Crystallogr. 2007, 40, 658-674. 
Insert Table of Contents artwork here

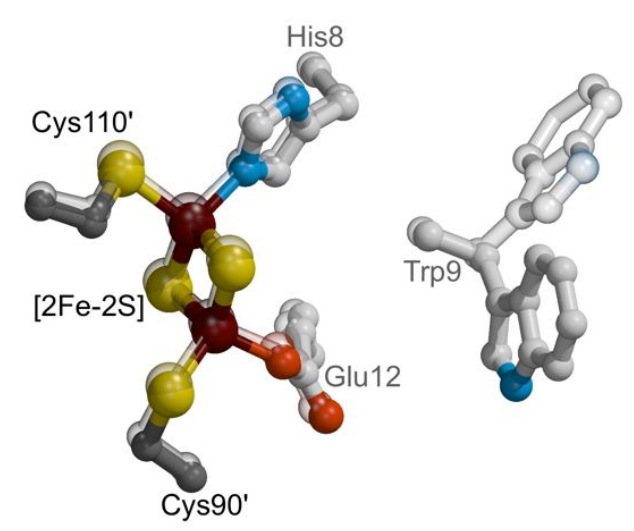

